\title{
GPS double difference statistics: with and without using satellite geometry
}

\author{
P. J. G. Teunissen \\ Delft Geodetic Computing Centre (LGR), Faculty of Geodesy, Delft University of Technology, Thijsseweg 11, \\ 2629 JA Delft, The Netherlands
}

Received 1 March 1996; Accepted 22 July 1996

\begin{abstract}
In this contribution GPS statistics are presented for the case that the relative receiver-satellite geometry is included in the single baseline model and for the case that the relative receiver-satellite geometry is excluded. It is shown that the statistics are linked through a particular form of a phased adjustment. Based on the stepwise approach of a phased adjustment, the impact of using satellite geometry or dispensing with it, on the least-squares estimators, on the teststatistics and their associated reliability, and on the integer ambiguity estimation, is presented and analyzed.
\end{abstract}

\section{Introduction}

Integer carrier phase ambiguity estimation plays a prominant role in many high precision relative GPS applications. Of the many different approaches proposed for integer ambiguity estimation, there are two which in particular have drawn much interest in the GPS literature. The two approaches differ in the model used for integer ambiguity estimation. In the first approach, which is the common mode of operation for most surveying applications, an explicit use is made of the available relative receiver-satellite geometry. It allows for instantaneous or almost instantaneous positioning, depending on whether both code and carrier phase data or only carrier phase data are used, see e.g. (Blewitt, 1989), (Frei and Beutler, 1990), (Hatch, 1991), (Wübbena, 1991), (Euler and Landau, 1992), (Teunissen, 1993) and (Tiberius and de Jonge, 1995). Integer ambiguity estimation is also possible however, when one opts for dispensing with the relative receiversatellite geometry, see e.g. (Hatch, 1982), (Euler and Goad, 1990), (Dedes and Goad, 1994), (Euler and Hatch, 1994) and (Teunissen, 1995a). In fact from a conceptual point of view, this is the simplest approach to integer ambiguity estimation. The code data are directly used to determine the unknown integer ambiguities of the observed phase data.
Interestingly enough, the above two approaches have been discussed and analyzed up to now without showing their connection. It is the purpose of this contribution to show and explicitly formulate this connection. As a consequence the two approaches can be cast in one framework, which has the additional advantage that the impact on the various stages of the data processing of using satellite geometry or dispersing with it, can be made clear.

Three topics will be addressed in this contribution. They are the least- squares adjustment, the statistical testing and associated reliability, and the integer ambiguity estimation. Starting from a decomposition of the single baseline model in section 2, it is shown that the above mentioned two approaches are linked through a particular form of a phased adjustment. Based on this phased adjustment, the least-squares solutions of the two approaches are analyzed and compared in section 3. In section 4, statistical teststatistics are given for the case satellite geometry is excluded and for the case satellite geometry is included. Also the reliability of these teststatistics is presented and compared. Finally, section 5 addresses the problem of integer ambiguity estimation for the two approaches.

\section{A decomposition of the single baseline model}

In this section we will introduce the two models that will form the basis of our study. They are the single baseline model with satellite geometry included and with satellite geometry excluded. It will be assumed that the separation between the two GPS receivers is such that the DD observables are sufficiently insensitive to orbital uncertainties in the fixed orbits and to residual ionospheric and tropospheric delays. It will also be assumed that during the observation time span, the same number of $m$ satellites are tracked. This assumption is realistic for relatively short time spans, which is the case when fast integer ambiguity estimation methods are applied. 


\subsection{Satellite geometry included}

We will start with the single baseline model which has the relative receiver-satellite geometry included. Based on the above assumptions its linear(ized) system of DD observation equations reads

$\left(I_{4} \otimes D^{T}\right) E\left\{y_{i}\right\}=\left(e_{4} \otimes D^{T} A_{i}\right) b+\left(C \otimes I_{m-1}\right) a$

with

$$
\begin{aligned}
y_{i} & =\left(\phi_{1}(i)^{T}, \phi_{2}(i)^{T}, p_{1}(i)^{T}, p_{2}(i)^{T}\right)^{T} \\
C & =\left(\lambda_{1} c_{1}, \lambda_{2} c_{2}\right) \\
a & =\left(a_{1}^{T}, a_{2}^{T}\right)^{T}
\end{aligned}
$$

for $i=1, \ldots, k . E\{$.$\} denotes the mathematical expecta-$ tion; $i$ denotes the epoch number and $k$ equals the total number of epochs; $\phi_{1}, \phi_{2}, p_{1}$ and $p_{2}$ are the $m$-vectors containing the (observed minus computed) metric single differenced (SD) phase and code observables on $L_{1}$ and $L_{2} ; D^{T}$ is the $(m-1) \times m$ DD matrix operator; $A_{i}$ is the $m \times 3$ SD design matrix that captures the relative receiver-satellite geometry at epoch $i ; b$ is the 3 -vector that contains the unknown increments of the three dimensional baseline; $\lambda_{1}$ and $\lambda_{2}$ are the wavelengths of $L_{1}$ and $L_{2} ;$ and, $a_{1}$ and $a_{2}$ are the two $(m-1)$-vectors that contain the unknown integer DD ambiguities.

In this contribution the unit matrix of order $p$ is denoted as $I_{p}$ and the $p$-vector having all ones as entries is denoted as $e_{p}$. Furthermore the canonical unit vector having the one as its $i$ th entry is denoted as $c_{i}$. The symbol ' $\otimes$ ' denotes the Kronecker product (sometimes also referred to as the direct product or tensor product). It is defined as

$M \otimes N=\left[\begin{array}{ccc}m_{11} N & \cdots & m_{1 q} N \\ \vdots & & \vdots \\ m_{p 1} N & \cdots & m_{p q} N\end{array}\right]$

where $M$ and $N$ are matrices, with $M=\left[m_{i j}\right]$, $i=1, \ldots, p, j=1, \ldots, q$. Since the Kronecker product will be used frequently in the sequel, we state here for easy reference some of its properties, see e.g. (Rao, 1973)

$$
\begin{aligned}
& (M \otimes N)^{T}=M^{T} \otimes N^{T} \\
& (M \otimes N)^{-1}=M^{-1} \otimes N^{-1} \\
& M_{1} M_{2} \otimes N_{1} N_{2}=\left(M_{1} \otimes N_{1}\right)\left(M_{2} \otimes N_{2}\right) \\
& \operatorname{rank}(M \otimes N)=\operatorname{rank}(M) \operatorname{rank}(N) \\
& \operatorname{vec} M N O=\left(O^{T} \otimes M\right) \operatorname{vec} N \\
& \operatorname{tr} L M N O=\left(\operatorname{vec} O^{T}\right)^{T}\left(N^{T} \otimes L\right) \operatorname{vec} M
\end{aligned}
$$

where $L, M, N$ and $O$ are matrices of appropriate order, where ' $t r$ ' denotes the trace and where 'vec' is the operator that transforms a matrix into a vector by stacking the columns of the matrix one underneath the other.

In the above description of the single baseline model we have taken the approach of explicitly showing the presence of the DD matrix operator. The reason for doing so, lies in the fact that the orthogonal projector that projects orthogonally onto the range space of the transposed DD matrix operator, plays an important role in our analysis. This projector can be represented in the following two ways

$D\left(D^{T} D\right)^{-1} D^{T}=I_{m}-\frac{1}{m} e_{m} e_{m}^{T}$

Note, since

$D X\left(X^{T} D^{T} D X\right)^{-1} X^{T} D^{T}=D\left(D^{T} D\right)^{-1} D^{T}$

for any $(m-1) \times(m-1)$ invertible matrix $X$, that the projector is independent of the choice of basis matrix in $R^{m-1}$ and thus also independent of the choice of reference satellite. Hence, for the projector it does not matter which satellite is chosen as reference. Since the projector projects orthogonally onto the space orthogonal to the range space of $D$, it will be denoted as $P_{e_{m}}^{\perp}$.

In our analysis we assume time correlation to be absent and the time-invariant variance matrix of the observables to be given as

$Q_{y_{i}}=\Sigma \otimes I_{m}$

with

$\Sigma^{-1}=\operatorname{diag}\left(\alpha_{1}, \alpha_{2}, \beta_{1}, \beta_{2}\right)$

Thus the $\alpha$ 's and the $\beta$ 's are the weights of the $L_{1}$ and $L_{2}$ phase and code observables. Hence, by setting them to zero, we can short-circuit the presence of any one of these four type of observables. This will become useful later in the sequel, since it will allow us to infer the impact of particular subsets of observables on our results.

The redundancy of the above given model depends on which type of observables are assumed present. Redundancy of a linear system of equations is defined as the number of equations minus the rank of the system's matrix. In case the system's matrix is of full rank, redundancy equals the number of equations minus the number of unknown parameters. Based on $k$ epochs, the redundancy $r$ reads

$$
\begin{array}{lll}
\phi_{1}, \phi_{2}, p_{1}, p_{2} & : r=2(2 k-1)(m-1)-3 \\
\phi_{1}, \phi_{2}, p_{1} & : r=(3 k-2)(m-1)-3 \\
\phi_{1}, p_{1} & : r=(2 k-1)(m-1)-3 \\
\phi_{1}, \phi_{2} & : r=2(k-1)(m-1)-3 \\
\phi_{1} & : r=(k-1)(m-1)-3
\end{array}
$$

This shows for example, that the phase-only situation requires a minimum of $k=2$ epochs, whereas if code data are added, a minimum of only one epoch is required. Also note that a minimum of $m=4$ satellites is needed, for these minimum number of epochs.

\subsection{Satellite geometry excluded}

The geometry of the relative receiver-satellite configuration at epoch $i$ is captured in the SD design matrix $A_{i}$. It contains the receiver-satellite line-of-sight vectors at epoch $i$. The presence of $A_{i}$ is due to the fact that model (1) is parametrized in terms of the baseline components. 
The matrix $A_{i}$ and thus also the relative receiver-satellite geometry, will be absent however, when instead of the baseline, the model is parametrized in terms of the receiver-satellite ranges. Thus in case the receiversatellite geometry is excluded, the linear system of DD observation equations reads

$\left(I_{4} \otimes D^{T}\right) E\left\{y_{i}\right\}=\left(e_{4} \otimes I_{m-1}\right) r_{i}+\left(C \otimes I_{m-1}\right) a$

for $i=1, \ldots, k$. The $(m-1)$-vector $r_{i}$ contains the DD ranges for epoch $i$. Note that no constraints in time are imposed on the $r_{i}$ 's. Thus the $r_{i}$ are time-variant, whereas the baseline vector $b$ in (1) is considered to be time-invariant. This implies that with model (5), the receivers need not to be stationary per se. Based on $k$ epochs, the redundancy of the above linear system reads

$$
\begin{array}{ll}
\phi_{1}, \phi_{2}, p_{1}, p_{2} & : r=(3 k-1)(m-1) \\
\phi_{1}, \phi_{2}, p_{1} & : r=2(k-1)(m-1) \\
\phi_{1}, p_{1} & : r=(k-1)(m-1) \\
\phi_{1}, \phi_{2} & : r=(k-2)(m-1)
\end{array}
$$

Note, that in contrast to model (1), code data is per se needed to be able to solve for the above model. But also note, that one can still have redundancy with code data absent. This happens for the dual frequency, phase-only case $\left(\phi_{1}, \phi_{2}\right)$. Hence in this particular case, statistical testing is still possible, despite the fact that the unknown parameters of the model can not be solved for.

\subsection{The link}

Upon comparing the above two models, (1) and (5), we note that the second model transforms into the first, once we include the geometric constraints

$r_{i}=\left(1 \otimes D^{T} A_{i}\right) b$

for $i=1, \ldots, k$. But this shows, that our first model can be solved in two steps, this in analogy to a phased adjustment. First, we solve (5). This gives $\hat{r}_{i}, i=1, \ldots, k$, and $\hat{a}$. With this result, an adjustment in a second step can be performed based on (7), which will give $\hat{b}$ and $\hat{\hat{a}}$. And the result of this second step will then be identical to the result one obtains when solving (1). Note that there are two types of constraints implicit in (7). First, if $m>4$, we have the constraints imposed by the relative receiver-satellite geometries at the individual epochs. But even when there is no satellite redundancy $(m=4)$, we still have the constraints that enter through the time-invariance of $b$.

\section{The least squares estimators}

In this section we will present the least-squares estimators of the two models (1) and (5), and study some of their characteristics. The approach followed will be based on a phased adjustment. We therefore commence solving for the single baseline model in which the relative receiver-satellite geometry is excluded.

\subsection{The first step}

Based on the single baseline model (5) and the variance matrix (3), the system of normal equations for $\hat{r}=\left(\hat{r}_{1}^{T}, \ldots, \hat{r}_{k}^{T}\right)^{T}$ and $\hat{a}=\left(\hat{a}_{1}^{T}, \hat{a}_{2}^{T}\right)^{T}$ reads

$\left[\begin{array}{cc}N_{r} & N_{r a} \\ N_{a r} & N_{a}\end{array}\right]\left[\begin{array}{l}\hat{r} \\ \hat{a}\end{array}\right]=\left[\begin{array}{l}n_{r} \\ n_{a}\end{array}\right]$

with

$$
\begin{aligned}
N_{r} & =\operatorname{tr} \Sigma^{-1}\left(I_{k} \otimes\left(D^{T} D\right)^{-1}\right) \\
N_{a r} & =\left(C^{T} \Sigma^{-1} e_{4} e_{k}^{T}\right) \otimes\left(D^{T} D\right)^{-1} \\
N_{a} & =k\left(C^{T} \Sigma^{-1} C\right) \otimes\left(D^{T} D\right)^{-1} \\
n_{r} & =\operatorname{vec}\left(\left(e_{4}^{T} \Sigma^{-1} \otimes\left(D^{T} D\right)^{-1} D^{T}\right) Y\right) \\
n_{a} & =k\left(C^{T} \Sigma^{-1} \otimes\left(D^{T} D\right)^{-1} D^{T}\right) \bar{y}
\end{aligned}
$$

and with the $m \times k$ matrix $Y=\left(y_{1}, \ldots, y_{k}\right)$ and the time average $\bar{y}=\frac{1}{k} \sum_{i=1}^{k} y_{i}$.

\section{The DD range vector $r$}

A top-down reduction of the above normal equations gives the reduced normal equations, from which $\hat{r}$ can be solved. The solution reads

$$
\hat{r}_{i}=D^{T}\left[\bar{p}_{w}+\frac{1}{1+\epsilon}\left(\left(\phi_{w}(i)-\bar{\phi}_{w}\right)+\epsilon\left(p_{w}(i)-\bar{p}_{w}\right)\right)\right]
$$

with the weighted and time averages

$$
\begin{array}{ll}
\phi_{w}(i)=\frac{\alpha_{1} \phi_{1}(i)+\alpha_{2} \phi_{2}(i)}{\alpha_{1}+\alpha_{2}} & \bar{\phi}_{w}=\frac{1}{k} \sum_{i=1}^{k} \phi_{w}(i) \\
p_{w}(i)=\frac{\beta_{1} p_{1}(i)+\beta_{2} p_{2}(i)}{\beta_{1}+\beta_{2}} & \bar{p}_{w}=\frac{1}{k} \sum_{i=1}^{k} p_{w}(i)
\end{array}
$$

where $\epsilon=\left(\beta_{1}+\beta_{2}\right) /\left(\alpha_{1}+\alpha_{2}\right)$. Thus the estimate of the range, equals the time average of the weighted average of the code data, plus a residual term that depends on the differences of the weighted averages of phase and code with their time averages. Note that these residual terms vanish in case the time average of $\hat{r}_{i}$ is taken.

Application of the error propagation law to (9) gives the variance-covariance matrix of $\hat{r}$ as

$Q_{\hat{r}}=\frac{1}{\alpha_{1}+\alpha_{2}} \frac{1}{1+\epsilon}\left(I_{k}+\frac{1}{\epsilon} P_{e_{k}}\right) \otimes\left(D^{T} D\right)$

Its inverse is the reduced normal matrix and reads

$N_{r}=\left(\alpha_{1}+\alpha_{2}\right)(1+\epsilon)\left(I_{k}-\frac{1}{1+\epsilon} P_{e_{k}}\right) \otimes\left(D^{T} D\right)^{-1}$

The projector $P_{e_{k}}$ projects orthogonally onto $e_{k}$.

Note that both matrices in the Kronecker product of the variance-covariance matrix are dense. The first matrix in this product determines the correlation between epochs. Hence, the correlation between $\hat{r}_{i}$ and $\hat{r}_{j}$, for $i \neq j$. The second matrix in the product determines the correlation between the satellite chan- 
nels; hence, between the entries of $\hat{r}_{i}$. This correlation is due to the double differencing process.

To see what happens to the correlation as $k$ gets larger, we consider the limit $k \rightarrow \infty$. Since $\lim _{k \rightarrow \infty}$ $P_{e_{k}}=0_{\infty}$, a zero matrix of infinite order, it follows from (10) that

$\lim _{k \rightarrow \infty} Q_{\hat{r}}=\frac{1}{\alpha_{1}+\alpha_{2}} \frac{1}{1+\epsilon} I_{\infty} \otimes\left(D^{T} D\right)$

Thus as $k$ gets larger the time correlation gets smaller and finally only the between-channel correlation remains. A somewhat similar situation occurs, if we assume the code-only situation

$\lim _{\alpha_{1}=\alpha_{2} \rightarrow 0} Q_{\hat{r}}=\frac{1}{\beta_{1}+\beta_{2}} I_{k} \otimes\left(D^{T} D\right)$

The phase-only situation however, gives a quite different result. Since

$\lim _{\beta_{1}=\beta_{2} \rightarrow 0} N_{r}=\left(\alpha_{1}+\alpha_{2}\right) P_{e_{k}}^{\perp} \otimes\left(D^{T} D\right)^{-1}$

it follows from the singularity of the projector that also the reduced normal matrix is singular. Thus code data are needed to solve for model (5).

Since phase data are far more precise than code data, $\epsilon$ will be small. We therefore have the approximation

$Q_{\hat{r}} \doteq\left(\frac{1}{\alpha_{1}+\alpha_{2}} I_{k}+\frac{1}{\beta_{1}+\beta_{2}} P_{e_{k}}\right) \otimes\left(D^{T} D\right)$

which shows that $Q_{\hat{r}}$ is dominated by the precision of the code data. In fact, if we assume the phase data to be exact, then

$\lim _{\alpha_{1}=\alpha_{2} \rightarrow \infty} Q_{\hat{r}}=\frac{1}{\beta_{1}+\beta_{2}} P_{e_{k}} \otimes\left(D^{T} D\right)$

which shows that now the variance-covariance matrix itself becomes singular. In this case, time differences of the $\hat{r}_{i}$ have zero variance.

\section{The DD ambiguity vector a}

A bottom-up reduction of the normal equations (8) allows us to solve for the DD ambiguity vector $\hat{a}=\left(\hat{a}_{1}^{T}, \hat{a}_{2}^{T}\right)^{T}$. It reads

$$
\begin{aligned}
& \hat{a}_{1}=\frac{1}{\lambda_{1}} D^{T}\left[\bar{\phi}_{1}-\bar{p}_{w}\right] \\
& \hat{a}_{2}=\frac{1}{\lambda_{2}} D^{T}\left[\bar{\phi}_{2}-\bar{p}_{w}\right]
\end{aligned}
$$

Thus the ambiguity estimates are simply scaled versions of the differences between the time average of the phase data and the weighted time average of the code data.

Application of the error propagation law gives

$Q_{\hat{a}}=Q \otimes D^{T} D$

with

$$
Q=\frac{1}{\lambda_{1} \lambda_{2} k\left(\beta_{1}+\beta_{2}\right)}\left[\begin{array}{cc}
\frac{\alpha_{1}+\beta_{1}+\beta_{2}}{\alpha_{1}} \frac{\lambda_{2}}{\lambda_{1}} & 1 \\
1 & \frac{\alpha_{2}+\beta_{1}+\beta_{2}}{\alpha_{2}} \frac{\lambda_{1}}{\lambda_{2}}
\end{array}\right]
$$

Note, since the precision of code data is in practice much poorer than that of phase data, that the variances of the ambiguities will be large if $k$ is too small. Hence, these variances can only be made smaller by taking a sufficient number of epochs into account. However, for integer estimation of the ambiguities it are not only the individual variances that count. When considering the ambiguity search space for integer ambiguity estimation, it is the complete variance covariance matrix that needs to be taken into account.

Matrix $Q_{\hat{a}}$ is the variance-covariance matrix of the complete $2(m-1)$ ambiguity vector $\hat{a}$. If attention is restricted however to the $(m-1)$ pairs of $L_{1}$ and $L_{2}$ ambiguities $\hat{a}_{1 i}, \hat{a}_{2 i} i=1, \ldots,(m-1)$, then it follows from (13) that their variance matrices are all the same and equal to $2 Q$. Thus if the correlation due to the double differencing process is neglected and integer ambiguity estimation is based on these individual pairs, then it is the matrix $Q$ which determines the unscaled ambiguity search space.

The following eigenvalue decomposition of (14) allows us to infer the shape and orientation of the ambiguity search space, which itself is a scaled version of the ambiguity confidence ellipse. Assuming that the phase data on the two frequencies are equally precise $\left(\alpha_{1}=\alpha_{2}=\alpha\right)$, we have

$Q=\left[\begin{array}{cc}\cos \theta & -\sin \theta \\ \sin \theta & \cos \theta\end{array}\right]\left[\begin{array}{cc}\mu_{1} & 0 \\ 0 & \mu_{2}\end{array}\right]\left[\begin{array}{cc}\cos \theta & \sin \theta \\ -\sin \theta & \cos \theta\end{array}\right]$

with

$\mu_{1,2}=\frac{\rho\left(\frac{\lambda_{2}}{\lambda_{1}}+\frac{\lambda_{1}}{\lambda_{2}}\right) \pm \sqrt{\left(\frac{\lambda_{2}}{\lambda_{1}}+\frac{\lambda_{1}}{\lambda_{2}}\right)^{2}+\left(\rho^{2}-1\right)\left(\frac{\lambda_{2}}{\lambda_{1}}-\frac{\lambda_{1}}{\lambda_{2}}\right)^{2}}}{2 \lambda_{1} \lambda_{2} k\left(\beta_{1}+\beta_{2}\right)}$

$\tan 2 \theta=2\left[\left(\frac{\lambda_{2}}{\lambda_{1}}-\frac{\lambda_{1}}{\lambda_{2}}\right) \rho\right]^{-1}$

where $\rho=\left(\alpha+\beta_{1}+\beta_{2}\right) / \alpha$. This result shows, since $\rho$ is close to one in practice, that the ambiguity search space is highly elongated $\left(\mu_{1} \gg \mu_{2}\right)$. Also note that the major axis of the ellipse will be oriented under an angle of somewhat less than 40 degrees with the coordinate axis of the $L_{1}$ ambiguity. The major axis will rotate clockwise if the precision of the code data gets better and/or when the precision of the phase data gets worse. An analytical study of the consequences of this particular shape and orientation of the ambiguity search space for integer least-squares ambiguity estimation, is given in (Teunissen, 1995a).

\subsection{The second step}

In this subsection we will perform the second phase of the adjustment. As a result the solution of the single 
baseline model (1) is obtained. In this second phase we have to solve for the model

$E\left\{\hat{r}_{i}\right\}=\left(1 \otimes D^{T} A_{i}\right) b$

for $i=1, \ldots, k$ using the variance matrix (10). The redundancy of this model equals $r=k(m-1)-3$ and it makes up for the difference in redundancy between (4) and (6).

\section{The baseline vector $b$}

Solving for (16), the least-squares estimate of the baseline vector follows as

$$
\begin{aligned}
\hat{b}= & W_{\hat{b}}^{-1} \times\left[\bar{A}^{T} P_{e_{k}}^{\perp} \bar{p}_{w}+\frac{1}{k \epsilon}\right. \\
& \left.\left(\sum_{i=1}^{k}\left(A_{i}-\bar{A}\right)^{T} P_{e_{k}}^{\perp}\left[\phi_{w}(i)+\epsilon p_{w}(i)\right]\right)\right]
\end{aligned}
$$

with variance matrix

$Q_{\hat{b}}=\frac{1}{k\left(\beta_{1}+\beta_{2}\right)} W_{\hat{b}}^{-1}$

where

$W_{\hat{b}}=\left[\bar{A}^{T} P_{e_{k}}^{\perp} \bar{A}+\frac{1+\epsilon}{k \epsilon} \sum_{i=1}^{k}\left(A_{i}-\bar{A}\right)^{T} P_{e_{k}}^{\perp}\left(A_{i}-\bar{A}\right)\right]$

and with the time averaged design matrix $\bar{A}=\frac{1}{k} \sum_{i=1}^{k} A_{i}$. Compare this result with (9). The above result clearly shows that the phase data only contributes to the precision of the baseline when there is a change of satellite geometry. In the absence of such a change, the baseline precision will be governed solely by the precision of the code data. Since the GPS relative receiver-satellite geometry changes only slowly in practice, a sufficiently long observation time span is needed to profit from the contribution of the phase data. This also stipulated the central role played by integer ambiguity estimation. When including the integer constraints $a \in Z^{2(m-1)}$, the phase data start to resemble very precise code data and therefore a drastic improvement in the baseline precision becomes feasible, even for short observation time spans.

\section{Updating the ambiguity vector a}

Note that the ambiguities are not involved in the observation equations of (16). Hence, our estimate $\hat{a}$ of the first phase does not contribute to the solution of the baseline vector. But the ambiguity vector $\hat{a}$ is correlated with $\hat{r}$. This implies therefore that the adjustment based on (16) does allow us to improve $\hat{a}$. With the least-squares residual vector $(\hat{r}-\hat{\hat{r}})$ of $(16)$, it follows that

$\hat{\hat{a}}=\hat{a}-Q_{\hat{a} \hat{r}} Q_{\hat{r}}^{-1}(\hat{r}-\hat{\hat{r}})$

Working this out, the update of the ambiguity vector is obtained as

$$
\begin{aligned}
& \hat{\hat{a}}_{1}=\hat{a}_{1}+\frac{1}{\lambda_{1}} D^{T}\left[\bar{p}_{w}-\bar{A} \hat{b}\right] \\
& \hat{\hat{a}}_{2}=\hat{a}_{2}+\frac{1}{\lambda_{2}} D^{T}\left[\bar{p}_{w}-\bar{A} \hat{b}\right]
\end{aligned}
$$

This result now clearly shows how the ambiguities obtained from the geometry-free model differ from the ambiguities obtained when the relative receiver-satellite geometry is taken into account. It shows that the time averaged weighted code data simply gets replaced by the time averaged least-squares range vector $\vec{A} \hat{b}$. In section 5 we will further discuss this difference in the context of integer least-squares ambiguity estimation.

\section{Test statistics and reliability}

In this section we will present teststatistics for both models (1) and (5). The measurement data will assumed to be normally distributed. First we will consider the overall model teststatistic. It allows one to detect departures from the assumptions underlying the model. Then we will consider two important teststatistics for identifying model errors. They are the cycleslip and the outlier teststatistic. The cycleslip teststatistic is considered for the phase data and the outlier teststatistic is considered for the code data. These teststatistics are optimal in the sense that they are uniformly most powerful and they have a standard normal distribution when the model is free from misspecifications.

We will also consider the reliability of the teststatistics. The internal reliability will be expressed by the minimal detectable biases (MDB). The MDB measures the size of the model error that can be detected with the teststatistic for a chosen detection probability and level of significance. As external reliability, we consider the impact of cycleslips and outliers of the size of the MDB's on the ambiguity estimates. This is particular of relevance in the context of integer ambiguity estimation. For the expressions of the teststatistics and their reliability measures, that hold for a general model of observation equations, we refer to (Baarda, 1968), (Teunissen, 1989).

\subsection{Overall model teststatistics}

Overall model teststatistics are given by the weighted residual sum of squares divided by the amount of redundancy. Under the assumption that the model has been specified correctly, they have a central Chi-squared distribution with the degrees of freedom being equal to the redundancy. The weighted residual sum of squares for model (5) reads

$\Omega=\sum_{j=1}^{2} \sum_{i=1}^{k} \omega_{i j}$

with

$$
\begin{aligned}
\omega_{i j}= & \alpha_{j}\left[\phi_{j}(i)-\bar{\phi}_{j}(i)-\delta(i)\right]^{T} P_{e_{m}}^{\perp}\left[\phi_{j}(i)-\bar{\phi}_{j}(i)-\delta(i)\right] \\
& +\beta_{j}\left[p_{j}(i)-\bar{p}_{w}(i)-\delta(i)\right]^{T} P_{e_{m}}^{\perp}\left[p_{j}(i)-\bar{p}_{w}(i)-\delta(i)\right]
\end{aligned}
$$


and

$\delta(i)=\frac{1}{1+\epsilon}\left[\left(\phi_{w}(i)-\bar{\phi}_{w}\right)+\epsilon\left(p_{w}(i)-\bar{p}_{w}\right)\right]$

Using (6) for the case of dual frequency code and phase, the overall model teststatistic reads therefore

$T_{1}=\frac{\Omega}{(3 k-1)(m-1)}$

Note that there are three types of averages involved in the above teststatistic. The weighted and time averages which we met earlier. But in addition there is also a satellite average involved. Since $P_{e_{m}}^{\perp}=I_{m}-P_{e_{m}}$, where $P_{e_{m}}$ projects orthogonally on the vector having all ones as entries, we see that also residuals are formed of the single differenced data with respect to their average over all $m$ satellite channels. This averaging is due to the double differencing process.

In order to obtain the overall model teststatistic of model (1), we first need the weighted residual sum of squares of $(16)$, It is given as $(\hat{r}-\hat{\hat{r}})^{T} Q_{\hat{r}}^{-1}(\hat{r}-\hat{\hat{r}})$ and when worked out, it reads as

$\Delta \Omega=\sum_{i=1}^{k} \Delta \omega_{i}$

with

$$
\begin{aligned}
\Delta \omega_{i}= & \left(\alpha_{1}+\alpha_{2}\right)\left[\left(A_{i}-\bar{A}\right) \hat{b}+\delta(i)\right]^{T} P_{e_{m}}^{\perp}\left[\left(A_{i}-\bar{A}\right) \hat{b}+\delta(i)\right] \\
& +\left(\beta_{1}+\beta_{2}\right)\left[\bar{p}_{w}-A_{i} \hat{b}+\delta(i)\right]^{T} P_{e_{m}}^{\perp}\left[\bar{p}_{w}-A_{i} \hat{b}+\delta(i)\right]
\end{aligned}
$$

As a consequence of the phased adjustment, the weighted residual sum of squares of model (1) simply follows from adding (22) to (20). The corresponding overall model teststatistic for the case satellite geometry is included, reads therefore

$T_{2}=\frac{\Omega+\Delta \Omega}{2(2 k-1)(m-1)-3}$

\subsection{The cycleslip teststatistic and its reliability}

In the following we present the teststatistics for identifying slips in the phase data. They will be given both for the case that satellite geometry is excluded as for the case that satellite geometry is included. For the cycleslip teststatistic we assume that a slip started to occur at epoch $l \leq k$ in the $L_{1}$ SD phase observable of the $i$ th satellite channel. The appropriate teststatistic for identifying such a model error reads then

$$
\begin{aligned}
& t_{1, s l i p}(i, l, k) \\
& =\sqrt{\alpha_{1} N \frac{m}{m-1} \frac{k}{l-1} \frac{c_{i}^{T} P_{e_{m}}^{\perp}\left[\tilde{\phi}_{1}-\bar{\phi}_{1}-\tilde{\delta}\right]}{\sqrt{1-\frac{\alpha_{1}}{\alpha_{1}+\alpha_{2}} \frac{1}{1+\epsilon}}}}
\end{aligned}
$$

with $N=k-l+1$ being the span between $l$ and $k$, and where the tilde . denotes the time average over this span. By replacing the $L_{1}$ phase data in (24) with the $L_{2}$ phase data and interchanging $\alpha_{1}$ and $\alpha_{2}$, one obtains the corresponding teststatistic for testing a slip in the $L_{2}$ phase data.
The MDB of the above cycleslip teststatistic, expressed in units of range rather than cycles, reads

$$
\begin{aligned}
& \left|\nabla_{1, s l i p}(i, l, k)\right| \\
& =\sqrt{\left[\frac{\lambda_{0}}{\alpha_{1}} \frac{m}{m-1} \frac{k}{(l-1) N}\right] /\left[1-\frac{\alpha_{1}}{\alpha_{1}+\alpha_{2}} \frac{1}{1+\epsilon}\right]}
\end{aligned}
$$

with $\lambda_{0}$ being the non-centrality parameter; it depends on the chosen detection probability and level of significance. With a probability of detection of 0.80 percent and level of significance of 0.001 , it follows that $\lambda_{0}=17.075$.

The above result clearly shows the four contributing factors to the cycleslip MDB. They are:

- $m$, the number of satellites used; The minimum number of satellites that can be used in the geometry-free situation, is of course $m=2$. But by using more satellites, smaller cycleslips can be detected.

- the time epochs $k, l$ and the span $N$; In order to consider the factor $k /(l-1) N$, we discriminate between the following three cases: $k$ is kept fixed, $N=k-l+1$ is kept fixed, or $l$ is kept fixed. With $k$ fixed, $(l-1) N / k$ becomes a parabola in $l$ having its maximum at $l=1+\frac{1}{2} k$. This shows that cycleslips are best detectable when they start in the middle of the time series and poorest detectable when they start at the beginning or at the end of the time series. Note that the MDB becomes infinite when $l=1$. In that case of course no cycleslip can be detected, since the slip gets absorbed by the corresponding ambiguity. With $N$ or $l$ fixed, the MDB gets smaller as $k$ gets larger. Thus as one would expect, smaller slips can be detected when more data are used.

- single frequency precision; If we assume that no phase data on the second frequency are available, then (25) reduces to

$$
\left|\nabla_{1, \text { slip }}(i, l, k)\right|=\sqrt{\frac{\lambda_{0}}{\alpha_{1}} \frac{m}{m-1} \frac{1+\epsilon}{\epsilon} \frac{k}{(l-1) N}}
$$

which shows that the MDB gets very large due to the poor precision of the code data ( $\epsilon$ is small). Thus, with single frequency data it is very difficult indeed to detect sufficiently small cycleslips. This situation can only be repaired by making $k$ large enough and having the slips start in the central region of the time series.

- precision of dual frequency data; if we assume that $\alpha_{1}=\alpha_{2}$ and recognize that the precision of the code data is much poorer than that of the phase data ( $\epsilon$ small), the MDB may be approximated as

$$
\left|\nabla_{1, s l i p}(i, l, k)\right| \doteq \sqrt{2 \frac{\lambda_{0}}{\alpha_{1}} \frac{m}{m-1} \frac{k}{(l-1) N}}
$$

which shows that in this case the MDB can become very small, even in the absence of code data. 
The above teststatistic (24) is optimal in terms of power. A somewhat simpler teststatistic, but one which is still close to being optimal, can be obtained when we make the approximations $\epsilon \doteq 0, \frac{1}{m} \doteq 0$ and set $\alpha_{1}=\alpha_{2}$. The corresponding cycleslip teststatistic can then be expressed as

$t_{1, s l i p}^{0}(i, l, k)=\sqrt{\frac{1}{2} \alpha_{1} \frac{(l-1) N}{k}} c_{i}^{T}\left[\left(\tilde{\phi}_{1}-\tilde{\phi}_{2}\right)-\left(\tilde{\tilde{\phi}}_{1}-\tilde{\tilde{\phi}}_{2}\right)\right]$

with the double tilde $\tilde{z}$ denoting the time average over the span 1 to $(l-1)$. Note that the residual in this expression now simply consists of a difference between the $L_{1}$ and $L_{2}$ phase data and the difference between two type of time averages. The first type of difference is needed to eliminate the SD receiver-satellite ranges and the second type of difference compares the data under the cycleslip regime with the data under the cycleslipfree regime. Also note, that the canonical unit vector $c_{i}$ now directly operates on the SD data. It simply selects the $i$ th entry from the SD residual vector.

It is gratifying to know that the geometry-free cycleslip teststatistic is already capable of detecting such small cycleslips in the phase data, even in the absence of code data, but provided that dual frequency data are used. The situation can of course only improve when satellite geometry is included. For the case that satellite geometry is included, we therefore only present the teststatistic and not its MDB. It reads

$$
\begin{aligned}
& t_{2, \text { slip }}(i, l, k)=\sqrt{\alpha_{1} N \frac{m}{m-1} \frac{k}{l-1}} \\
& \times \frac{c_{i}^{T} P_{e_{m}}^{\perp}\left[\left(\tilde{\phi}_{1}-\bar{\phi}_{1}\right)-(\tilde{A}-\bar{A}) \hat{b}\right]}{\sqrt{1-\alpha_{1} N \frac{m}{m-1} \frac{k}{l-1} c_{i}^{T} P_{e_{m}}^{\perp}(\tilde{A}-\bar{A})^{T} Q_{\hat{b}}(\tilde{A}-\bar{A}) P_{e_{m}}^{\perp} c_{i}}}
\end{aligned}
$$

\subsection{The outlier teststatistic and its reliability}

For the outlier teststatistic we again consider first the geometry-free situation. We assume that an outlier has occurred at the single epoch $l \leq k$ in the $L_{1} \mathrm{SD}$ code observable of the $i$ th satellite channel. The corresponding teststatistic for identifying such a model error reads then

$$
\begin{aligned}
& t_{1, \text { outlier }}(i, l, k) \\
& =\sqrt{\beta_{1 \frac{m}{m-1}}} \frac{c_{i}^{T} P_{e_{m}}^{\perp}\left[p_{1}(l)-\bar{p}_{w}-\delta(l)\right]}{\sqrt{1-\frac{\beta_{1}}{\beta_{1}+\beta_{2}} \frac{1}{1+\epsilon}\left(\epsilon+\frac{1}{k}\right)}}
\end{aligned}
$$

Simply replacing $p_{1}(l)$ by $p_{2}(l)$ and interchanging $\beta_{1}$ and $\beta_{2}$, gives the corresponding teststatistic for the $L_{2}$ code observable. Again, as in the cycleslip case, a somewhat less optimal, but simpler teststatistic can be obtained if we make the approximations $\epsilon \doteq 0, \frac{1}{m} \dot{=}$, set $\beta_{1}=\beta_{2}$ and neglect $\delta(l)$. It reads

$$
t_{1, \text { outlier }}^{0}(i, l, k)=\sqrt{\beta_{1} \frac{2 k}{2 k-1}} c_{i}^{T}\left[p_{1}(l)-\bar{p}_{w}\right]
$$

The MDB of the outlier teststatistic (27) reads

$$
\begin{aligned}
& \left|\nabla_{1, \text { outlier }}(i, l, k)\right| \\
& \quad=\sqrt{\left[\frac{\lambda_{0}}{\beta_{1}} \frac{m}{m-1}\right] /\left[1-\frac{\beta_{1}}{\beta_{1}+\beta_{2}} \frac{1}{1+\epsilon}\left(\epsilon+\frac{1}{k}\right)\right]}
\end{aligned}
$$

This result shows that the outlier MDB is quite large and that the contribution of the phase data in lowering the MDB is marginal. In fact we have the bound

$$
\left|\nabla_{1, \text { outlier }}(i, l, k)\right|>\sqrt{\frac{\lambda_{0}}{\beta_{1}} \frac{m}{m-1}}
$$

This shows, when the power and level of significance are set at 0.80 resp. 0.001 , that for two satellites, the outlier MDB is larger than 0.8 resp. 2.4 meters for an undifferenced code precision of 30 resp. 10 centimetres. For ten satellites, this would give 0.6 resp. 1.8 metres. For a larger power even larger MDB values would be obtained. The large value of the outlier MDB is a potentially dangerous situation, in particular with respect to the integer leastsquares ambiguity estimation. It is therefore of interest to consider the external reliability of the above MDB and to infer its impact on the least-squares estimate of the ambiguities. Using (12), the impact on each one of the entries of the $L_{1}$ ambiguity vector reads

$$
\left|c_{j}^{T} \nabla \hat{a}_{1}\right|=\frac{1}{k \lambda_{1}} \frac{\beta_{1}}{\beta_{1}+\beta_{2}} c_{j}^{T} D^{T} c_{i}\left|\nabla_{1, \text { outlier }}(i, l, k)\right|
$$

for $j=1, \ldots,(m-1)$. For the $L_{2}$ ambiguity vector a similar expression can be given. Substitution of (28) and using $\left(c_{j}^{T} D^{T} c_{i}\right)^{2} \leq c_{j}^{T} D^{T} D c_{j} c_{i}^{T} c_{i}=2$ with $\beta_{1}=\beta_{2}, \epsilon \dot{=}$, gives

$\left|c_{j}^{T} \nabla \hat{a}_{1}\right| \leq \frac{1}{\lambda_{1}} \sqrt{\frac{\lambda_{0}}{\beta_{1}} \frac{m}{m-1} \frac{1}{k(2 k-1)}}$

for $j=1, \ldots,(m-1)$. This shows that the impact on the entries of the ambiguity vector is approximately proportional to $1 / k$. Hence, by using a sufficient number of epochs, the impact can be made arbitrarily small. However, the main idea of integer least-squares ambiguity estimation is to be able to estimate and validate the integer ambiguities in the shortest time span possible. For $k=15, m=10$ and a precision of the undifferenced code data of $30 \mathrm{~cm}$, the above bound on all entries of the ambiguity vector equals about $0.5 \mathrm{mtr}$. This shows that a strenghtening of the model so as to be able to detect smaller outliers, would be very welcome indeed. We therefore now consider the outlier teststatistic and its MDB for the case satellite geometry is included. The teststatistic reads

$$
\begin{aligned}
& t_{2, \text { outlier }}(i, l, k) \\
& =\sqrt{\beta_{1 \frac{m}{m-1}}} \frac{c_{i}^{T} P_{e_{m}}^{\perp}\left[p_{1}(l)-A_{l} \hat{b}\right]}{\sqrt{1-\beta_{1} \frac{m}{m-1} c_{i}^{T} P_{e_{m}}^{\perp} A_{l} Q_{\hat{b}} A_{l}^{T} P_{e_{m}}^{\perp} c_{i}}}
\end{aligned}
$$

and its MDB is given as 


$$
\begin{aligned}
& \left|\nabla_{2, \text { outlier }}(i, l, k)\right| \\
& \quad=\sqrt{\left[\frac{\lambda_{0}}{\beta_{1}} \frac{m}{m-1}\right] /\left[1-\beta_{1} \frac{m}{m-1} c_{i}^{T} P_{e_{m}}^{\perp} A_{l} Q_{\hat{b}} A_{l}^{T} P_{e_{m}}^{\perp} c_{i}\right]}
\end{aligned}
$$

Analogous to above we now infer the MDB's impact on the least-squares ambiguities. With $\beta_{1}=\beta_{2}, Q_{\hat{b}} \leq$ $\left[\bar{A}^{T} P_{e_{m}}^{\perp} \bar{A}\right]^{-1} / 2 k \beta_{1}$ and

$c_{i}^{T} P_{e_{m}}^{\perp} \bar{A}\left[\bar{A}^{T} P_{e_{m}}^{\perp} \bar{A}\right]^{-1} \bar{A}^{T} P_{e_{m}}^{\perp} c_{i}=\frac{m-1}{m} \sin ^{2} \theta$

where $\theta$ is the angle between $c_{i}$ and the orthogonal complement of the range space of the matrix $\left(e_{m}, \bar{A}\right)$, and the approximation $A_{l} \doteq \bar{A}$, we now get for all entries of the updated ambiguity vector

$\left|c_{j}^{T} \nabla \hat{\hat{a}}_{1}\right| \leq \frac{1}{\lambda_{1}} \sqrt{\frac{\lambda_{0}}{\beta_{1}} \frac{\sin ^{2} \theta}{k\left(2 k-\sin ^{2} \theta\right)}}$

for $j=1, \ldots,(m-1)$. In this result, we now clearly see the impact of satellite geometry at work through the presence of the $\sin \theta$. We have a large impact when $c_{i}$ can be written as a linear combination of the columns of the matrix $\left(e_{m}, \bar{A}\right)$ and a zero impact when $c_{i}$ is orthogonal to the columns of this matrix. Compare (33) with (30).

\section{Integer least-squares ambiguity estimation}

The purpose of integer ambiguity estimation is to be able, via the inclusion of the integer constraints on the ambiguities, to obtain a drastic improvement in the precision of the baseline solution. Denoting the integer least-squares estimate of the ambiguity vector as $\check{a}$ and the corresponding baseline vectors as $\check{b}$, the baseline solution becomes $\check{b}=\hat{b}-Q_{\hat{b} \hat{\hat{a}}} Q_{\hat{\hat{a}}}^{-1}(\hat{\hat{a}}-\check{a})$. The baseline vectors $\hat{b}$ and $\check{b}$ are usually referred to as the 'float' resp 'fixed' baselines. Application of the error propagation law shows for short observation time spans, if we assume $\check{a}$ to be nonstochastic, that $Q_{\check{b}} \ll Q_{\hat{b}}$.

Integer least-squares ambiguity estimation in case satellite geometry is excluded, amounts to solving the minimization problem

$\min _{a}(\hat{a}-a)^{T} Q_{\hat{a}}^{-1}(\hat{a}-a) \quad$ with $a \in Z^{2(m-1)}$

For the case satellite geometry is included, $\hat{a}$ needs to be replaced by $\hat{\hat{a}}$ and $Q_{\hat{a}}$ by $Q_{\hat{\hat{a}}}$. In order to solve the above minimization problem, its objective function is used to introduce an ellipsoidal region in $R^{2(m-1)}$

$(\hat{a}-a)^{T} Q_{\hat{a}}^{-1}(\hat{a}-a) \leq \chi^{2}$

This region, referred to as the ambiguity search space, is then used to set up a search for the minimizer of (34). The search space is centred at $\hat{a} \in R^{2(m-1)}$, its orientation and elongation are governed by the ambiguity variance matrix $Q_{\hat{a}}$, and its size can be controlled through the selection of the positive constant $\chi^{2}$. In (Teunissen,
1993), the least-squares ambiguity decorrelation adjustment (LAMBDA) method was introduced for efficiently solving (34). The method consists of two steps: (a) a sequential conditional least-squares adjustment on the ambiguities; and (b) an ambiguity transformation that allows the ambiguities to become largely decorrelated. The purpose of the sequential conditional least-squares adjustment is to be able to write the quadratic form of (35) as a sum of independent squares. This sum allows us then to write (35) as

$\left(\hat{a}_{i \mid I}-a_{i}\right)^{2} \leq \sigma_{\hat{a}_{i \mid I}}^{2}\left[\chi^{2}-\sum_{j=1}^{i-1} \frac{\left(\hat{a}_{j \mid J}-a_{j}\right)^{2}}{\sigma_{\hat{a}_{j \mid J}}^{2}}\right]$

for $i=1, \ldots, 2(m-1)$. Here the shorthand notation $\hat{a}_{j \mid J}$ has been used for the conditional least-squares ambiguity estimate $\hat{a}_{j \mid(j-1), \ldots, 1}$. The above bounds can be used to set up a search for the minimizer of (34). In case of short observation time spans however, this search is seriously hindered by the presence of a large discontinuity in the spectrum of DD ambiguity conditional variances $\sigma_{\hat{a}_{i \mid z}}^{2}$. Various numerical examples of the discontinuity are given in (Teunissen et al., 1994). The spectrum can however be flattened and lowered by means of the decorrelating ambiguity transformation. As a result a new set of ambiguities is obtained, with highly precise and largely uncorrelated ambiguities, and for which the search based on (36) can now be performed in an efficient manner. For more details on the LAMBDA-method, we refer to e.g (Teunissen, 1993), (Teunissen, 1995b). The implementation aspects of the method are presented in detail in (Jonge, de and Tiberius, 1996).

Since the impact of the decorrelating ambiguity transformation depends on the amount of discontinuity in the spectrum of conditional variances with respect to its unconditional counterpart, it is of interest to understand the signature of this spectrum. In this section, the signature of the spectrum will be discussed in a qualitative sense, both for the case satellite geometry is excluded as well as for the case satellite geometry is included.

\subsection{The DD ambiguity search space with satellite geometry excluded}

In this subsection we will study the spectrum of conditional variances of the ambiguity variance matrix (13). For the purpose of this subsection it is more convenient however, to work with an ordering of the ambiguities that differs from the one which has been used so far. Instead of using the ambiguity vector $a=\left(a_{1}^{T}, a_{2}^{T}\right)^{T}$, in which first all $L_{1}$ ambiguities are collected followed by the $L_{2}$ ambiguities, we will now use an ordering in which the $L_{1}$ and $L_{2}$ ambiguities are treated as pairs. Hence, we define the reordered ambiguity vector as $N=\left(N_{1}^{T}, \ldots, N_{m-1}^{T}\right)^{T}$, with $N_{i}=\left(a_{1 i}, a_{2 i}^{T}\right)$ for $i=1, \ldots,(m-1)$. This reordering implies a simple swapping of the two matrices in the Kronecker product of (13). Thus the variance matrix of the reordered least-squares ambiguity vector $\hat{N}$ reads 
$Q_{\hat{N}}=D^{T} D \otimes Q$

In (Teunissen, 1993), it was shown that a sequential conditional least-squares adjustment is mathematically equivalent to performing an $L D L^{T}$-decomposition on the variance matrix. Since the ambiguity variance matrix equals a Kronecker product in our case, we first need to know how the $L D L^{T}$-decomposition performs on this Kronecker product. Let

$A_{0}=A_{1} \otimes A_{2}$

where $A_{0}, A_{1}$ and $A_{2}$ are three symmetric matrices of appropriate order. Furthermore, let

$A_{0}=L_{0} D_{0} L_{0}^{T}, A_{1}=L_{1} D_{1} L_{1}^{T}$ and $A_{2}=L_{2} D_{2} L_{2}^{T}$

be their $L D L^{T}$-decompositions. It follows then from the properties of the Kronecker product that

$L_{0}=L_{1} \otimes L_{2}$ and $D_{0}=D_{1} \otimes D_{2}$

Hence, in order to obtain the $L D L^{T}$-decomposition of the Kronecker product we can concentrate on the $L D L^{T}$ decompositions of the individual matrix entries in the product itself.

\section{The $L D L^{T}$-decomposition of $D^{T} D$ and $Q$}

We will start with the $L D L^{T}$-decomposition of the matrix $D^{T} D$, which will be denoted as

$D^{T} D=L_{1} D_{1} L_{1}^{T}$

Since the matrix $D^{T} D$ has 2's on its diagonal and 1's on all its off-diagonals, it is not difficult to verify that $L_{1}$ and $D_{1}$ are given as

$$
\left\{\begin{array}{c}
L_{1}=\left[\begin{array}{ccccc}
1 & & & & \\
\frac{1}{2} & 1 & & & \\
\frac{1}{2} & \frac{1}{3} & 1 & & \\
\vdots & \vdots & \vdots & \ddots & \\
\frac{1}{2} & \frac{1}{3} & \frac{1}{4} & \cdots & 1
\end{array}\right] \\
D_{1}=\operatorname{diag}\left(2, \frac{3}{2}, \cdots, \frac{m}{m-1}\right)
\end{array}\right.
$$

We also need their inverses. They are given as

$$
\left\{\begin{array}{c}
L_{1}^{-1}=\left[\begin{array}{ccccc}
1 & & & & \\
-\frac{1}{2} & 1 & & & \\
-\frac{1}{3} & -\frac{1}{3} & 1 & & \\
\vdots & \vdots & \vdots & \ddots & \\
-\frac{1}{m-1} & -\frac{1}{m-1} & -\frac{1}{m-1} & \cdots & 1
\end{array}\right] \\
D_{1}^{-1}=\operatorname{diag}\left(\frac{1}{2}, \frac{2}{3}, \cdots, \frac{m-1}{m}\right)
\end{array}\right.
$$

Also the factors of the $L D L^{T}$ - decomposition of matrix $Q$ in (14)

$Q=L_{2} D_{2} L_{2}^{T}$

are readily given. They read

$$
\left\{\begin{aligned}
L_{2}= & {\left[\begin{array}{cc}
1 & 0 \\
\frac{\alpha_{1}}{\alpha_{1}+\beta_{1}+\beta_{2}} \frac{\lambda_{1}}{\lambda_{2}} & 1
\end{array}\right] } \\
D_{2}= & \operatorname{diag}\left(\frac{1}{k \lambda_{1}^{2}}\left(\frac{1}{\alpha_{1}}+\frac{1}{\beta_{1}+\beta_{2}}\right)\right. \\
& \left.\frac{1}{k \lambda_{2}^{2}}\left(\frac{1}{\alpha_{2}}+\frac{1}{\alpha_{1}+\beta_{1}+\beta_{2}}\right)\right)
\end{aligned}\right.
$$

The sequential search bounds

We are now ready to study the characteristics of the ambiguity search space

$(\hat{N}-N)^{T} Q_{\hat{N}}^{-1}(\hat{N}-N) \leq \chi^{2}$

and its corresponding search bounds. Starting with the $L D L^{T}$-decomposition of $D^{T} D$, we have for the inverse of the ambiguity variance matrix

$$
\begin{aligned}
Q_{\hat{N}}^{-1} & =\left(L_{1} D_{1} L_{1}^{T} \otimes Q\right)^{-1} \\
& =\left(L_{1}^{-T} \otimes I_{2}\right)\left(D_{1}^{-1} \otimes Q^{-1}\right)\left(L_{1}^{-1} \otimes I_{2}\right)
\end{aligned}
$$

Hence

$$
\begin{aligned}
(\hat{N}-N)^{T} & Q_{\hat{N}}^{-1}(\hat{N}-N) \\
= & \left(\left(L_{1}^{-1} \otimes I_{2}\right)(\hat{N}-N)\right)^{T}\left(D_{1}^{-1} \otimes Q^{-1}\right) \\
& \times\left(\left(L_{1}^{-1} \otimes I_{2}\right)(\hat{N}-N)\right)
\end{aligned}
$$

Since the $L D L^{T}$-decomposition has the statistical interpretation of a sequential conditional least-squares adjustment, we have

$\left(L_{1}^{-1} \otimes I_{2}\right)(\hat{N}-N)=\left(\hat{N}_{c}-N\right)$

with

$\hat{N}_{c}=\left(\hat{N}_{1}^{T}, \hat{N}_{2 \mid 1}^{T}, \ldots, \hat{N}_{m-1 \mid M-1}^{T}\right)^{T}$

And because of the special structure of the triangular factor $L_{1}^{-1}$, we have

$\hat{N}_{i \mid I}=\left(\hat{N}_{i}-N_{i}\right)-\frac{1}{i} \sum_{j=1}^{i-1}\left(\hat{N}_{j}-N_{j}\right)$

for $i=1, \ldots,(m-1)$. It follows therefore from (44), (45) and (46) that the ambiguity search space (43) may also be written as

$\sum_{i=1}^{m-1} \frac{i}{i+1}\left(\hat{N}_{i \mid I}-N_{i}\right)^{T} Q^{-1}\left(\hat{N}_{i \mid I}-N_{i}\right) \leq \chi^{2}$

This expression now allows us to set up the sequential two dimensional bounds 


$$
\left\{\begin{array}{c}
\left\|\hat{N}_{1}-N_{1}\right\|^{2} \leq 2 \chi^{2} \\
\left\|\hat{N}_{2 \mid 1}-N_{2}\right\|^{2} \leq \frac{3}{2}\left[\chi^{2}-\left\|\hat{N}_{1}-N_{1}\right\|^{2}\right] \\
\vdots \\
\left\|\hat{N}_{j \mid J}-N_{j}\right\|^{2} \leq \frac{j+1}{j}\left[\chi^{2}-\sum_{i=1}^{j-1} \frac{i}{i+1}\left\|\hat{N}_{i \mid I}-N_{i}\right\|^{2}\right]
\end{array}\right.
$$

for up to $j=m-1$. The norm taken is with respect to the $Q$-metric. Note that this set of bounds can be visualized in $R^{2}$ as a set of $(m-1)$ number of ellipses, all having the same shape, but centred at different locations $\hat{N}_{j \mid J}$, with a size that reduces as $j$ gets larger.

In order to pursue the search, we of course still have to express the above two-dimensional quadratic forms as sum of squares. But since all ellipses have the same shape, this can be done in one step. Denoting the upper bounds of (48) as $\bar{\chi}_{j}^{2}$, the two scalar bounds for the ellipses

$$
\left\|\hat{N}_{j \mid J}-N_{j}\right\|^{2} \leq \bar{\chi}_{j}^{2}
$$

follow from using (42) as

$$
\left\{\begin{array}{c}
\left(\hat{N}_{j \mid J, 1}-N_{j, 1}\right)^{2} \leq \frac{1}{k \lambda_{1}^{2}}\left(\frac{1}{\alpha_{1}}+\frac{1}{\beta_{1}+\beta_{2}}\right) \bar{\chi}_{j}^{2} \\
\left(\hat{N}_{j|J, 2| 1}-N_{j, 2}\right)^{2} \\
\leq \frac{1}{k \lambda_{2}^{2}}\left(\frac{1}{\alpha_{2}}+\frac{1}{\alpha_{1}+\beta_{1}+\beta_{2}}\right)\left[\bar{\chi}_{j}^{2}-k \lambda_{1}^{2} \frac{\left(\hat{N}_{j \mid J, 1}-N_{j, 1}\right)^{2}}{\frac{1}{\alpha_{1}}+\frac{1}{\beta_{1}+\beta_{2}}}\right]
\end{array}\right.
$$

with

$\hat{N}_{j|J, 2| 1}=\hat{N}_{j \mid J, 2}-\frac{\lambda_{1}}{\lambda_{2}} \frac{\alpha_{2}}{\alpha_{1}+\beta_{1}+\beta_{2}}\left(\hat{N}_{j \mid J, 1}-N_{j, 1}\right)$

The two sets (48) and (49) now provide for the complete $2(m-1)$ scalar bounds which can be used for the search of the integer least-squares ambiguities. Due to the Kronecker product in (37), the spectrum of conditional variances of $Q_{\hat{a}}$ breaks up in two parts. One part that is determined by the entries of the diagonal matrix $D_{1}$ and a second part that is determined by the entries of the diagonal matrix $D_{2}$. The entries of $D_{1}$ do not show a discontinuity; they get smaller rather smoothly. The two entries of $D_{2}$ however, do show a large difference. The second entry is for all practical purposes far smaller than the first entry. This implies that the search from ellipse to ellipse in (48) can be executed rather efficiently, but that the search within each ellipse based on (49) will exhibit the problem of search halting. This result shows that in out pursuit of a decorrelating ambiguity transformation, we only need to consider matrix $Q$ and not the matrix $D^{T} D$. Thus if $Z^{T}$ denotes the $2(m-1) \times 2(m-1)$ decorrelating ambiguity transformation, it will be of the form

$Z^{T}=Z_{2}^{T} \otimes I_{m-1}$

and transform the ambiguity variance matrix as

$Q_{\hat{z}}=Z^{T} Q_{\hat{a}} Z=Z_{2}^{T} Q Z_{2} \otimes D^{T} D$

How the LAMBDA-method constructs matrix $Z_{2}$ has been shown analytically in (Teunissen, 1995a).

\subsection{On the spectrum of ambiguity variance matrices}

In the previous subsection it was found that the spectrum of conditional variances of the ambiguity variance matrix $Q_{\hat{a}}$ could be seen to consist of two parts. The first part decreased rather smoothly in size, but the second part contained a rather large discontinuity. As it was pointed out, it is this large discontinuity that forms a hindrance for the efficient search of the integer leastsquares ambiguities. That is, if the search is performed on the original DD ambiguities. In the LAMBDAmethod however, transformed ambiguities are used, which have a flattened spectrum. The ability of the method to flatten the spectrum depends on the existence of the discontinuity in the spectrum of the original DD ambiguities. Hence, although the discontinuity in the original spectrum hinders the DD ambiguity search, it at the same time enables the LAMBDA-method to come up with a lower and flattened spectrum. And this transformed spectrum will be lower, when there are more DD ambiguities that have a small conditional variance. In this subsection we will show under which circumstances one can expect to have a discontinuity in the spectrum of DD ambiguity conditional variances. This then at the same time shows in a qualitative sense the ability of the LAMBDA-method to come up with precise transformed ambiguities.

In order to understand the origin of the discontinuity, we will first rewrite matrix $Q$ of (13) as a particular sum of two matrices

$Q=\frac{1}{k\left(\beta_{1}+\beta_{2}\right)}\left[\epsilon Q_{1}+Q_{2}\right]$

with

$Q_{1}=\frac{1}{\lambda_{1} \lambda_{2}}\left[\begin{array}{cc}\frac{\alpha_{1}+\alpha_{2}}{\alpha_{1}} \frac{\lambda_{2}}{\lambda_{1}} & 0 \\ 0 & \frac{\alpha_{1}+\alpha_{2}}{\alpha_{2}} \frac{\lambda_{1}}{\lambda_{2}}\end{array}\right]$

and

$Q_{2}=\frac{1}{\lambda_{1} \lambda_{2}}\left[\begin{array}{c}\sqrt{\frac{\lambda_{2}}{\lambda_{1}}} \\ \sqrt{\frac{\lambda_{1}}{\lambda_{2}}}\end{array}\right]\left[\begin{array}{c}\sqrt{\frac{\lambda_{2}}{\lambda_{1}}} \\ \sqrt{\frac{\lambda_{1}}{\lambda_{2}}}\end{array}\right]^{T}$

This decomposition shows that matrix $Q$ equals the sum of a full rank matrix (even a diagonal matrix) and a rank defect matrix (rank defect equals one) of which the entries are significantly larger than those of the full rank matrix. We will now show that any variance matrix that can be written as such a sum, will have conditional variances that are significantly smaller than their unconditional counterparts. Let $V$ be a variance matrix of order $m$, that can be written as

$V=v W+X X^{T}$

in which $v$ is very small, $W$ is of full rank and $X$ is of full rank, having $n<m$ columns. Let

$V=\left[\begin{array}{ll}V_{11} & V_{12} \\ V_{21} & V_{22}\end{array}\right]$ 
be its partitioning, in which the diagonal blocks are of order $n<m$ resp. $(m-n)$. Matrices $W$ and $X$ are partitioned accordingly. The variance matrix of the last $(m-n)$ elements reads then

$V_{22}=v W_{22}+X_{2} X_{2}^{T}$

When a least-squares adjustment is carried out in which a conditioning on the first $n$ elements is applied, then the corresponding conditioned variance matrix will read

$V_{22 \mid 1}=\left(-V_{21} V_{11}^{-1}, I_{m-n}\right) V\left(-V_{21} V_{11}^{-1}, I_{m-n}\right)^{T}$

Since $v$ is very small by assumption, we may approximate $V_{21} V_{11}^{-1}$ in the above expression by $X_{2} X_{1}^{-1}$. This gives then the approximation

$V_{22 \mid 1} \doteq v\left(-X_{2} X_{1}^{-1}, I_{m-n}\right) V\left(-X_{2} X_{1}^{-1}, I_{m-n}\right)^{T}$

since $\left(-X_{2} X_{1}^{-1}, I_{m-n}\right) X=0$. If we now compare (52) with (53), we observe the interesting fact that where $v$ was only a scale factor of the first matrix in the sum of the unconditioned variance matrix, it has become a scale factor of the complete matrix in the conditioned case. Hence, the entries of the conditioned matrix are significantly smaller than those of its unconditioned counterpart. This shows that one can indeed expect a discontinuity in the spectrum if variance matrices are structured like (51).

We are now in a position to discuss the structure of the variance matrices of the ambiguities. Starting with the case that satellite geometry is excluded, we have

$Q_{\hat{a}}=\frac{1}{k\left(\beta_{1}+\beta_{2}\right)}\left[\epsilon\left(Q_{1} \otimes D^{T} D\right)+\left(Q_{2} \otimes D^{T} D\right)\right]$

This is a matrix of order $2(m-1)$ of which the first matrix in the sum is of full rank. The entries of the first matrix are however significantly smaller than those of the second matrix, which is if rank $(m-1)$. Hence, this second matrix has a rank defect of $(m-1)$, which shows that the $(m-1)$ out of the $2(m-1)$ conditional variances will be small. This is indeed in agreement with our results of the previous subsection.

The above holds for the dual frequency case. If we consider the single frequency case, then the ambiguity variance matrix can be written as

$Q_{\hat{a}_{1}}=\frac{1}{k \beta_{1}}\left[\epsilon_{1}\left(\frac{1}{\lambda_{1}^{2}} \otimes D^{T} D\right)+\left(\frac{1}{\lambda_{1}^{2}} \otimes D^{T} D\right)\right]$

with $\epsilon_{1}=\beta_{1} / \alpha_{1}$. Now the second matrix in the sum fails to have a rank defect. Hence, no discontinuity in the spectrum will occur and no lowering of the spectrum together with a return of transformed ambiguities with a high precision can be expected. This thus makes quite clear what role is played by dual frequency data.

We will now consider the case when satellite geometry is included. Application of the error propagation law to (19) gives

$Q_{\hat{a}}=\left[\frac{1}{k\left(\alpha_{1}+\alpha_{2}\right)}\left(Q_{1} \otimes D^{T} D\right)+\left(Q_{2} \otimes D^{T} \bar{A} Q_{\hat{b}} \bar{A}^{T} D\right)\right]$
A comparison with (54) shows that the inclusion of satellite geometry resulted in a replacement of the full rank matrix $D^{T} D / k\left(\beta_{1}+\beta_{2}\right)$ with the rank defect matrix $D^{T} \bar{A} Q_{\hat{b}} \bar{A}^{T} D$. Thus the variance matrix (56) has a rank defect which now stems from both $Q_{2}$ and $D^{T} \bar{A} Q_{\hat{b}} \bar{A}^{T} D$. Since the rank defect of the second matrix in the sum of (56) equals $2(m-1)-3$, the spectrum will have as many small conditional variances.

In the single frequency case, we have

$Q_{\hat{\hat{a}}_{1}}=\left[\frac{1}{k \alpha_{1}}\left(\frac{1}{\lambda_{1}^{2}} \otimes D^{T} D\right)+\left(\frac{1}{\lambda_{1}^{2}} \otimes D^{T} \bar{A} Q_{\hat{b}} \bar{A}^{T} D\right)\right]$

The rank defect of the second matrix now equals $(m-4)$, as opposed to zero in case satellite geometry is excluded. This thus also makes quite clear what role is played by satellite redundancy. Having only four satellites, with single frequency data will thus give a spectrum in which no small conditional variances are to be expected.

\section{Summary}

In this contribution it has been shown that the geometry-free GPS adjustment statistics are linked by means of a phased adjustment to their geometry dependent counterparts. This link enabled us to study the impact of satellite geometry on the least-squares estimators, on the teststatistics and on the ambiguity search spaces. The receiver-satellite range vector $r$ and ambiguity vector $a$ are the unknown parameters in case the geometry is excluded. When the geometry is included however, the unknown parameters are the baseline vector $b$ and the ambiguity vector $a$.

As a result of the adjustment of the geometry-free model, one obtains the least-squares estimates and corresponding variance covariance matrices

$\left[\begin{array}{l}\hat{r} \\ \hat{a}\end{array}\right], \quad\left[\begin{array}{ll}Q_{\hat{r}} & Q_{\hat{r} \hat{a}} \\ Q_{\hat{a} \hat{r}} & Q_{\hat{a}}\end{array}\right]$

This is the result of the first step. Combining it in a leastsquares sense with the constraints

$r_{i}=\left(1 \otimes D^{T} A_{i}\right) b$

gives the result of the second step

$\left[\begin{array}{c}\hat{b} \\ \hat{a}\end{array}\right], \quad\left[\begin{array}{cc}Q_{\hat{b}} & Q_{\hat{b} \hat{a}} \\ Q_{\hat{a} \hat{b}} & Q_{\hat{a}}\end{array}\right]$

And this solution is identical to the least-squares solution of the model when the receiver-satellite geometry is included.

Following the two steps in the phased adjustment, teststatistics were given for overall model validation, cycleslip detection and outlier detection. These teststatistics are optimal in the sense of their power of detection. Also some simpler, but slightly less optimal teststatistics were given. The internal reliability of the teststatistics was discussed in terms of their minimal detectable biases (MDB). For the geometry-free case, 
the dual frequency cycle slip MDB was shown to be small in general. The outlier MDB however, was shown to be quite large. Also the external reliability of the outlier teststatistic, as measured by the impact on the ambiguities, was shown to be quite large, thus posing a potential threat to the estimation and validation of the integer ambiguities. With satellite geometry included however, the outlier MDB can be pulled down to a more acceptable value.

Integer ambiguity estimation is feasible both when satellite geometry is excluded as well as when it is included. Although the two approaches differ in the model on which they are based, there is no conceptual difference in how the integer ambiguities can be estimated. In both cases the integer estimation is based on the (real-valued) least-squares ambiguities and their variance covariance matrix. Thus in case of the geometry-free model it is based on $\hat{a}$ and $Q_{\hat{a}}$, in case the geometry is included, it is based on $\hat{\hat{a}}$ and $Q_{\hat{\hat{a}}}$. The LAMBDA-method can be applied to both cases. For the geometry-free case, the DD ambiguity search space together with the corresponding search bounds, was described analytically. We also stressed the importance of the signature of the spectrum of ambiguity conditional variances for an efficient search. Despite the difference in model used, it was shown that all ambiguity variance matrices have one important property in common. They all can be written as a sum of two matrices of which the first is of full rank and the second is possibly rank defect having entries which are large compared to those of the first matrix. It was shown that the rank defect of the second matrix is instrumental for determining the signature of the spectrum. Since this rank defect is directly coupled to the presence or absence of satellite geometry and to the type of data used, the role played by satellite redundancy and dual frequency data in flattening and lowering the spectrum when transforming the ambiguities, has been made clear.

Acknowledgement. This work was supported by the Alexander von Humboldt Stiftung and written during the author's stay at the University of Stuttgart (Germany), with professor E.W. Grafarend as his host.

\section{References}

Baarda, W. (1968): A Testing Procedure for use in Geodetic Networks, Netherlands Geodetic Commission, New Series, Vol. 2, No. 4.
Blewitt, G. (1989): Carrier phase ambiguity resolution for the Global Positioning System applied to geodetic baselines up to 2000 km. Journal of Geophysical Research, Vol. 94, No. B8, pp. 10.187-10.203.

Dedes, G., C. Goad (1994): Real-time cm-level GPS Positioning of Cutting Blade and Earth Movement Equipment. Proceedings of the 1994 National Technical Meeting ION, San Diego, California, pp. 587-594.

Euler, H.-J., C. Goad (1990): On Optimal Filtering of GPS Dual Frequency Observations without using Orbit Information. Bulletin Geodesique, Vol. 65, pp. 130-143.

Euler, H.-J., H. Landau (1992): Fast GPS Ambiguity Resolution On-The-Fly for Real-time Applications. Proceedings 6th Int, Geod. Symp. on Satellite Positioning. Columbus, Ohio, 17-20 March 1992, pp. 650-729.

Euler, H.-J.,R. Hatch (1994): Comparison of Several AROF Kinematic Techniques. Proceedings ION GPS-94, Salt Lake City, Utah, USA, Sept. 20-23, 1994, pp. 363-370.

Frei, E., G. Beutler (1990): Rapid Static Positioning Based on the Fast Ambiguity Resolution Approach FARA: Theory and First Results. Manuscripta Geodaetica, Vol. 15, No. 6, 1990.

Hatch, R. (1982): The Synergism of GPS Code and Carrier Measurements. Proceedings 3th International Geod. Symp. on Satellite Positioning, Las Cruces, New Mexico, 8-12 February, 1982, Vol. 2, pp. 1213-1231.

Hatch, R. (1991): Instantaneous Ambiguity Resolution, Proceedings of the IAG Int. Symp. 107 on Kinematic Systems in Geodesy, Surveying and Remote Sensing, Sept. 10-13, 1990, SpringerVerlag, pp. 299-308.

Jonge, de P. J., C. C. J. M. Tiberius (1996): The LAMBDA method for integer ambiguity estimation: implementation aspects. Delft Geodetic Computing Centre, LGR-Series, No. 12.

Teunissen, P. J. G. (1989): Introduction to Testing Theory. Delft Geodetic Computing Centre, 142 p.

Teunissen, P. J. G. (1993): Least-Squares Estimation of the Integer GPS ambiguities. Invited Lecture, Section IV Theory and Methodology, IAG General Meeting, Beijing, China, August 1993. Also in LGR-Series No. 6, 16 p.

Teunissen, P. J. G., P.J. de Jonge, C.C.J.M. Tiberius (1994): On the Spectrum of the GPS DD-Ambiguities. Proceedings ION GPS94, Salt Lake City, Utah, USA, Sept. 20-23, 1994, pp. 115-124.

Teunissen, P. J. G. (1995a): An analytical study of ambiguity decorrelation using dual frequency code and carrier phase. Journal of Geodesy, Vol, 70, pp.515-528.

Teunissen, P. J. G. (1995b): The Least-Squares Ambiguity Decorrelation Adjustment: A Method for Fast GPS Integer Ambiguity Estimation. Journal of Geodesy, Vol. 70, No. 1/2.

Tiberius, C. C. J. M., P. J. de Jonge (1995): Fast Positioning using the LAMBDA-method. Proceedings DSNS95, April 24-28, Bergen, Norway.

Rao, C. R. (1973): Linear Statistical Inference and its Applications, Wiley, New York.

Wübbena, G. (1991): Zur Modellierung von GPS Beobachtungen für die Hochgenaue Positions-bestimmung. Universität Hannover, Germany. 